\title{
Knowledge Representation in Virtual Teams: A Perspective Approach for Synthetic Worlds
}

\author{
Giacomo Fiumara $^{1}$, Dario Maggiorini ${ }^{2}$, Alessandro Provetti ${ }^{1,3}$, \\ and Laura A. Ripamonti ${ }^{2}$ \\ ${ }^{1}$ Dip. di Fisica, Sezione d'Informatica. Università degli Studi di Messina \\ V.le F. Stagno d'Alcontres 31, I-98166 Messina, Italy \\ \{gfiumara, ale\} @unime.it \\ ${ }^{2}$ Dip. d'Informatica e Comunicazione, Università degli Studi di Milano \\ Via Comelico, 39, I-20135, Milan, Italy \\ \{dario, ripamonti\}@dico.unimi.it \\ ${ }^{3}$ Oxford-Man Institute, University of Oxford \\ Eagle House, Walton Well Rd., Oxford OX2 6ED, UK
}

\begin{abstract}
Thanks to ICT, new organizational forms are emerging that keep the pace of an increasingly demanding competitive environment. Among them, Virtual Enterprises (VEs) and Virtual Teams (VTs) represent two challenging organizational forms from the point of view of effective management.

An open issue in VEs/VTs is the social and economical sustainability of knowledge sharing in virtual environments, which is often underestimated. We address it from the point of view of "virtual worlds" and developed a prototypal solution that facilitates the cooperative building/sharing of knowledge representation.
\end{abstract}

Keywords: Virtual worlds, Virtual teams, Ontologies, Folksonomies.

\section{Introduction}

Barriers against effective integration among people belonging to different organizational functions/organizations are a well known phenomenon, whose complexity grows with the degree of virtualization of the organization: this problem becomes particularly relevant when "virtual enterprises" (VEs) and/or "virtual teams" (VTs) are involved. Barriers to integration proceed from socio-cultural misalignment among people belonging to different organizational environments, and they could be classified into five main classes (personality, cultural, language, organizational and physical barriers [1]). Integration among different organizational units has been often addressed through the integration of information systems, achieved through the use of appropriate software applications. Anyway, this approach leads to failure in many cases, since it solves only part of the problem [2]: good interaction among different functions/people can be achieved only when a good (social) interaction pattern is established among all the actors involved. This means that not only data and information systems should be integrated, but also - and perhaps mainly - social systems and social networks. This issue could be looked at as a problem of sustainability, For the purpose of this work, we adopt a description of sustainability which puts sustainable 
systems at the intersection of social, economic and environmental sustainability. In particular, we will put the accent on the interplay between the first two subtypes of sustainability (nevertheless, we underline that virtual organizations may positively impact on the environmental problems due, e.g., to a lesser need for trips and physical meetings [3]). The economic sustainability of an effective VE/VT is intertwined with its social (from the perspective of its members) sustainability. In particular, we claim that the problem of social sustainability can be tackled through the lenses of a semiotic ladder [4], which offers us a key to understand how to unfold integration into its different components (physical, empiric, syntactic, semantic, pragmatic and social), as shown in Fig.1.

This paper is based on the theoretical assumptions derived in [26], that highlight how a sound and appropriate support to the higher levels of the semiotic ladder could be achieved through a perspective approach - rooted into both the socio-semantic web [5] and the pragmatic web [6] paradigms - which couples formal and social interaction in virtual worlds. In particular, Multi User Virtual Environments (MUVEs) are considered suitable environments for supplying digital habitats to VEs/VTs which are sustainable both from a social and economic point of view. The idea is to combine the social capabilities of the current web2.0 applications with those of the semantic web in representing, managing, and retrieving explicit knowledge, within an immersive virtual world which extends and augments the actual one.

Building on these bases, we describe the characteristics of a prototypal implementation of a tool for creating three-dimensional representations of knowledge in virtual worlds (see also [7]).

\section{Three Paradigms for Sharing Knowledge and Social Interaction}

We can define a VT as "a temporary arrangement of individuals belonging to different organizations and cultures, possessing different functional backgrounds, and working across different time zones on a common task" ([7], p. 61). This definition well describes how VTs are structured, nevertheless it is worth noting that the key factor for the existence of VTs is the possibility to rely on an effective communication system, based on digital technologies. This aspect has been too long underestimated, perhaps also due to the fact that digital-based infrastructures are more and more perceived as commodities, forgetting the increasingly relevant social implications of their use. And, as a consequence, forgetting that effective interaction among different organizational units must rely upon communication and not only on the mere sharing of data and information.

To exploit effectively the opportunities offered by ICTs as enabler of integration, it is of fundamental importance to understand how ICTs can support communication, social interaction and knowledge generation and sharing among people, also and mainly when they become the principal (if not the only) means of interaction among the actors of the organization. Form this standpoint, three different paradigms have different pros and contras, as shown in Fig. 1 (for an exhaustive discussion on this topic, see: [3]):

- Semantic Web (SW) offers powerful tools for managing and retrieving explicit knowledge (i.e., ontologies, semantic search tools, etc.), while underestimates the aspects linked to social interaction and tacit knowledge management. Moreover, knowledge representations cannot be modified, updated or integrated with the 
degree of agility and flexibility needed by virtual organizations. This paradigm could be used to address at most the semantic level of the semiotic ladder.

- Web2.0 (and Pragmatic web - [6]) is the expression of the social use of the web. Through tools for sharing knowledge and information on a social basis (e.g., social tagging and folksonomies), it adds agility, flexibility, and portability to knowledge representations. Anyway, it has a number of drawbacks; such as the lacking of semantic tools for searching, the impossibility to create complex knowledge maps, etc. Moreover, the web2.0 is a mesh-up of different technologies, information sources, web sites, etc., thus lacking one specific point of aggregation able to become the "environment" for building relations supporting integration. Again, this paradigm is unable to support effectively enough the last two levels of the semiotic ladder, while - compared to SW - it could enhance the support to the semantic level.

- $\quad$ Multi User Virtual Environments (MUVEs) are exceptional for augmenting actual lives of their users [8]. They support effectively the extension of people actual social networks and they can be moulded into places that may become synthetic loci for sharing tacit knowledge and expertise (see, e.g. the SUN OpenWonderland Project). MUVEs can be exploited as synthetic environments for supporting the higher levels of the semiotic ladder (pragmatic and social world), hence they can be of help in lowering certain integration barriers. Unfortunately, at the moment, even the more developed MUVEs (i.e., Second Life - SL) are quite lacking from the point of view of formalized systems for collecting, sharing, retrieving and reusing knowledge (even in its explicit form). Again this approach alone is not enough.

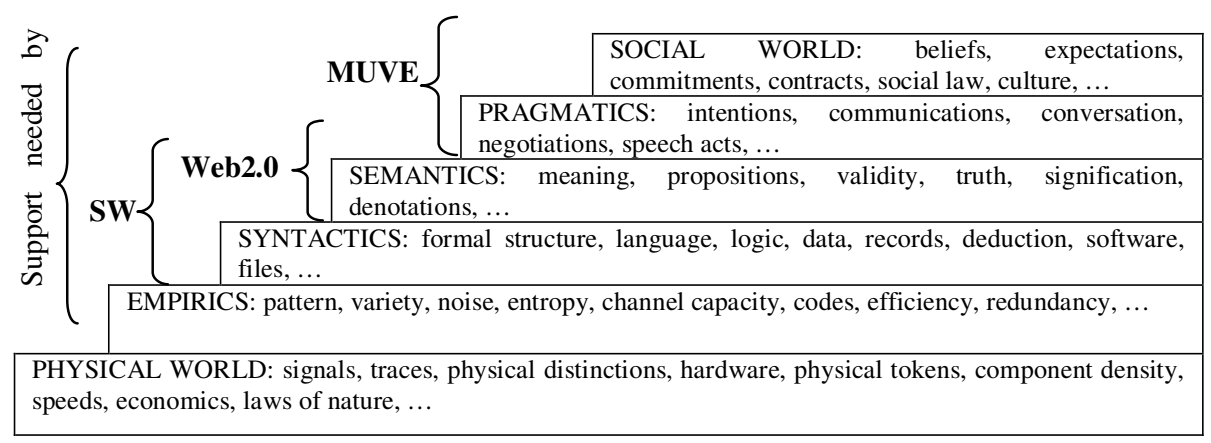

Fig. 1. Support offered by SW, Web2.0 and MUVEs to the "semiotic ladder"

Hence, none of these paradigms - when adopted alone - seems to be a solution good enough to address effectively the problem of integration.

\section{Towards Collaboratively Built, Shared, 3D Representations of Knowledge}

Since its appearance, SL attracted the attention of researchers and practitioners, and several have been the experiments aiming at coupling virtual environments with 
semantic web applications running on external web servers [9], [10], [11], [12], [13], and [14]; anyway, none of them seems to put the accent on the fundamental importance of the social factor in the process of sharing knowledge. On the other hand, several organizations (e.g., IBM, SUN, Northrop Grumman Corporation, etc.) are experiencing with SL as an environment for team building, but without coupling it with any semantic software application.

\subsection{Representing Knowledge in a MUVE}

In our project, we have designed and implemented a software application that brings into the virtual world the organization of a folksonomy, intended as a keywordsdeployed user annotation. The folksonomy is located outside SL and represented in the standard RDF notation (thus maximizing flexibility and accessibility by nonhuman agents), but it can be updated also by users (avatars and/or agents) from within SL. Hence, our solution blurs the distinction between consulting/annotating objects inside and outside SL and the distinction between user and agent annotations. The application is potentially available to all authorized avatars approaching its 3D representation. The conceptual representation can evolve through collaborative concepts' annotation and updating, thus making a shared semantic to emerge. From a strictly technical point of view, the long-term goal of our project is the creation of a knowledge base within SL that is accessible both to human users (through their avatars) and software applications. Our knowledge base will support automated access, selection, and consultation of the objects appearing on one or more specified SL loci.

\subsection{Composition of the Knowledge Base: The SCOT Ontology}

A Knowledge Base (KB) can be seen as a set of formulae that express information (knowledge) about an object or a resource which is to be described. By now, almost all semantics annotations on the web are expressed by means of RDF (Resource Description Framework). At the very bare, the attributes of a resource are described by means of properties which in turn are described within ontologies, which are publicly available through the web.

In order to describe the composition of declarative languages used in our application, we have adopted the SCOT (Semantic Cloud of Tags) ontology [15], [16]. We briefly recall the properties of the SCOT ontology that were used more frequently in our application:

- TagCloud. This class describes the main repository of the tagging activity, namely the cloud of tags. All other properties derive from this one.

- HasTag. This property describes the set of tags associated to an object. This property belongs to another ontology, namely SIOC (Semantically-Interlinked Online Communities).

- Tag. This class describes the single tag belonging to a cloud of tags. It is a string used to identify a specified resource.

- OwnAFrequency is a RDF DataTypeProperty used to describe the absolute frequency of occurrences of a tag; fore in detail, the total number of times the specified tag containing this property has been associated to an object.

These properties have a hierarchical structure: TagCloud contains HasTag which in turn contains Tag, which contains OwnAFrequency. 


\subsection{Taxonomies and Folksonomies}

A taxonomy defines both a hierarchical classification of concepts and the complex of ideas and rules with which object are classified. The taxonomic model seems to be in contrast with the folksonomic model, as in the former there is a well-founded rule to bind concepts while in the latter there is no rule at all. In this application we created a hybrid model which exploits hierarchical features of taxonomic model and the lack of rules of folksonomic model. In short, we created a folksonomy organized as a taxonomy. The only relationship established between concepts is that of inheritance between a concept and its ancestor. Since no other relationship is expresses, not even ordering among siblings to a common ancestor, the tree format is natural and sufficiently expressive. Of course, the scalability of this model is somewhat limited since the tagging of all conceivable concepts would produce a graph rather than a tree. Nevertheless, the goal of the model is the creation of a topic map, with an arbitrary depth, derived from a single concept.

\subsection{Bridging the Gap between 3D Conceptual Maps and Web-Based Folksonomies}

The main result of the application is a KB with semantic models. The KB resides in a RDF native repository, and is reachable by the application within SL and/or HTTP protocols and/or plain TCP/IP connections. The database, being multi-protocol, allows for the content to be usable in different contexts, assuring the stability and availability of data from within different applications developed in environments different from SL. An high-level architecture of our application is depicted in Fig. 2. Our application works as follows: associated to the 3D objects in SL there are scripts coded in LSL, the scripting language available in SL. When an avatar interacts with any of them one of the following actions takes place: a query to the RDF repository located outside SL, a tagging action of a resource, or the representation of the KB. Queries coded in SPARQL, the RDF query language, can be submitted from avatars as well as from users in the real world.

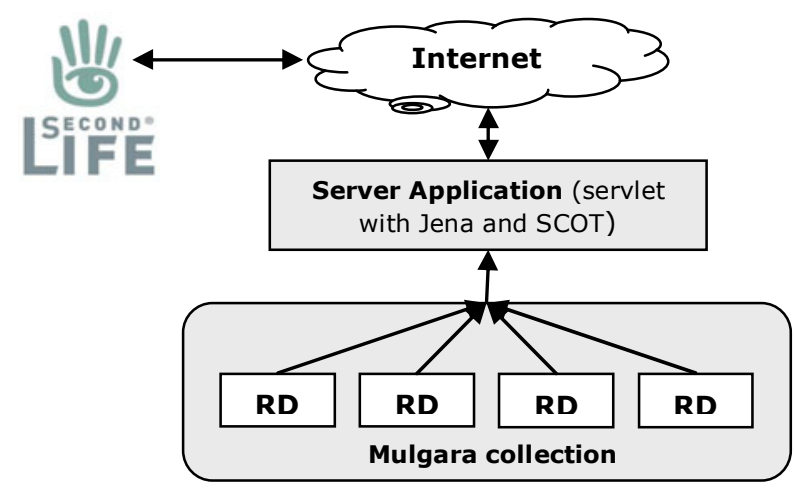

Fig. 2. Schema of the application 
The same tool allows to build multi-level topic maps. Topic maps can cover great lengths (w.r.t. Second Life virtual land), and may be composed by a large number of 3D objects; hence, the client application must be activated in locations where suitable resources are available. In order to preserve performances of the hosting server, topic maps undergo an automatic self-destruction after a timeout. The prototype of our application has been tested for a period of three months against a varying number of avatars. The response time and the scalability seem encouraging, even if estimating the performances of this application is not easy, as they depend on various factors. Among these factors the most relevant are the degree of network congestion, the load on servers, the number of 3D objects to manage, and the complexity of the submitted query.

\section{Conclusions and Future Development}

The virtualization of teams/organizations may negatively affect the quality of interaction and knowledge sharing among individuals, thus generating problems of socioeconomical sustainability. VEs/VTs may exploit opportunities offered by ICT (that should be considered a critical success factor) as enablers of social interaction and knowledge sharing to partially overcome those constraints. To achieve this goal, it is necessary to deploy solutions able to build trust and empathy, improve and develop social relations, increase opportunities to interact, reflect interdependencies of tasks, formalize knowledge representation, bridge long distances, and create (in)formal meeting places. The major part of these requirements could be addressed through a socio-technical approach, aimed at designing solutions and applications able to foster knowledge sharing into digital social environments. At the moment the principal approaches to this problem (i.e., SW, Web2.0) are too focused on one or another of the problem's facets, lacking a holistic vision. In particular, they lack in supporting effectively online (synthetic) extension of people identity and relations, as well as the creation of synthetic "places" where social networks can live and evolve. These latter aspects of synthetic life can instead flourish in synthetic worlds (MUVEs), which are unable to offer a convincing solution for managing knowledge in a formalized way. To overcome those limits, we have presented a perspective approach that intersects SW, Web2.0 and MUVEs, aiming at providing tools for sharing knowledge in a synthetic social environment.

\section{References}

1. Vandevelde, A., Van Dierdonck, R.: Managing the design-manufacturing interface. International Journal of Operations \& Production Management 23(11), 1326-1348 (2003)

2. Tan, A., Kondoz, A.M.: Barriers to virtual collaboration. In: CHI 2008 Extended Abstracts on Human Factors in Computing Systems, CHI 2008, Florence, Italy, April 5-10, pp. 2045-2052. ACM, New York (2008), http: / /doi.acm.org/10.1145/1358628.1358636

3. Ripamonti, L.A., Peraboni, C.: Managing the Design-Manufacturing Interface in VEs through MUVEs: a perspective approach. In: Putnik, G. (ed.) International Journal of Computer Integrated Manufacturing, Special issue: "Semiotics based integrated manufacturing". Taylor \& Francis, Abington (forthcoming, 2010) ISSN: 0951-192X 
4. Stamper, R.K.: Signs, information, norms and systems. In: Holmqvist, B., et al. (eds.) Signs at work. De Gruyter, Berlin (1996)

5. Cahier, J.P., Zacklad, M.: Socio-Semantic Web applications: towards a methodology based on the Theory of the Communities of Action. In: COOP 2004 Workshop on Knowledge Interaction and Knowledge Management (2004)

6. Schoop, M., de Moor, A., Dietz, J.L.G.: The Pragmatic Web: a Manifesto. Communication of the ACM 49(5), 75-76 (2006)

7. Kahai, S.S., Carroll, E., Jestice, R.: Team Collaboration in Virtual Worlds. The Data Base for advances in Information Systems 28(4) (November 2007)

8. Boellstorff, T.: Coming of Age in Second Life. An Anthropologist Explores the Virtually Human. Princeton University Press, Princeton (2008)

9. Bogdanovych, A., Rodriguez, J.A., Simoff, S.J., Sierra, A.C.C.: Developing virtual heritage applications as normative multiagent systems. In: AOSE. LNCS. Springer, Heidelberg (2009)

10. Galanis, D., Karakatsiotis, G., Lampouras, G., Androutsopoulos, I.: Naturalowl: Generating texts from OWL ontologies in Protégé and in Second Life. In: 18th European Conference on Artificial Intelligence (2008)

11. Galanis, D., Karakatsiotis, G., Lampouras, G., Androutsopoulos, I.: An open-source natural language generator for OWL ontologies and its use in Protegé and Second Life. In: EACL (Demos), pp. 17-20. The Association for Computer Linguistics (2009)

12. Kleinermann, F., Troyer, O.D., Creelle, C., Pellens, B.: Adding semantic annotations, navigation paths and tour guides to existing virtual environments. In: Wyeld, T.G., Kenderdine, S., Docherty, M. (eds.) VSMM 2007. LNCS, vol. 4820, pp. 100-111. Springer, Heidelberg (2008)

13. Schneider, M., Kratzer, F., Mainzer, K.: SEMSL: Tagging and data linking for Second Life. In: 7th International Semantic Web Conference - ISWC 2008 (2008),

http: / /data.semanticweb.org/conference/iswc/2008/paper /

poster_demo/76 (Retrieved 2010-03-26)

14. Oberlander, J., Karakatsiotis, G., Isard, A., Androutsopoulos, I., Unit, D.C.: Building an adaptive museum gallery in second life (2008),

http://nlp.cs.aueb.gr/pubs/mw2008_preprint.pdf

(Retrieved 2010-03-26)

15. Kim, H.L., Passant, A., Breslin, J., Scerri, S., Decker, S.: Review and Alignmnet of Tag Ontologies for Semantically-Linked Data in Collaborative Tagging Spaces. In: Proceedings of the 2nd International Conference on Semantic Computing, San Francisco, USA (2008)

16. Kim, H.L., Scerri, S., Breslin, J., Decker, S., Kim, H.G.: The State of the Art in Tag Ontologies: A Semantic Model for Tagging and Folksonomies. In: Proceedings of the Int. Conference on Dublin Core and Metadata Applications, Berlin, Germany (2008) 